\title{
Bisphenol S Promotes the Progression of Prostate Cancer by Regulating the Expression of COL1A1 and COL1A2.
}

shengdi Liu

nanjing medical university

Hua Li ( $\square$ lihua1123333@163.com )

\section{Research Article}

Keywords: BPS, Prostate cancer, Genetic comprehension

Posted Date: April 8th, 2022

DOl: https://doi.org/10.21203/rs.3.rs-808288/v3

License: (c) (i) This work is licensed under a Creative Commons Attribution 4.0 International License. Read Full License

Version of Record: A version of this preprint was published at Toxicology on April 1st, 2022. See the published version at https://doi.org/10.1016/j.tox.2022.153178. 


\section{Abstract}

In recent decades, Bisphenol S (BPS), which have been considered as alternatives for Bisphenol A (BPA), have become widely used in personal care products, paper products and food. Clarifying the relationship between bisphenol and tumors is of great significance for the treatment and prevention of diseases. In this work, we discovered a new method to predict the correlation between bisphenol interactive genes and tumors. The transcriptome profile and interactive genes of bisphenol were obtained from the Cancer Genome Atlas and Genotype-Tissue Expression, Comparative Toxicology Genomics and PharmMapper database. Gene Ontology and Kyoto Encyclopedia of Genes and Genomes analysis showed that interactive genes were mainly enriched in prostate cancer. Gene targetd prediction and gene set variation analysis proved that bisphenol exert potential effects on prostate cancer. The operating characteristic curves and survival analysis showed that role of COL1A1 and COL1A2 in predicting the prognosis of prostate cancer. Cell counting kit-8 assay demonstrated that the cells with BPS-treated could remarkably promote the cell proliferation ability in both PC-3 and LNCap cells. Wound healing assay and the transwell assay demonstrated that the cells with BPS-treated could significantly promote the cell invasion capacity of prostate cells. Two key genes, COL1A1 and COL1A2, were significantly upregulated with BPStreated in the PC-3 and LNCap cells.

\section{Introduction}

In the past few decades, with urbanization and industrialization levels continue to rise, environmental pollution has become a worldwide problem. Endocrine disrupting chemicals (EDCs), which can be found in agriculture and industry, have been proved to affect hormone synthesis, metabolism and function(Ma et al.2019). Bisphenol, as a widely used EDC, can be found in plastics, receipts, food packaging, and other products. regulate hormone signal paths and various other biological functions in a wide range of products (Murata et al. 2017). Relevant studies have shown that EDs can interact with some endocrine signaling pathways, such as estrogen receptor (Legeay et al. 2017). Estrogen receptor can regulate estrogen synthesis and related enzyme activities by transcription factors. Classical estrogen receptors include two subtypes of ERa and ERß, both of which have similar structures. ERa is mainly expressed in hormone-targeted tissues, such as breast, uterus, testes and ovaries. On the contrary, ER $\beta$ is mainly expressed in the prostate, bladder, lung and intestine (Rochester et al. 2015).

In the past 40 years, the incidence and mortality of tumors have been increasing. As is estimated by the American Cancer Society, more than 1.7 million new cancers will be diagnosed in 2019, and there will be approximately 607,000 cancer-related deaths (Giri et al. 2020). Prostate cancer is one of the most common malignant tumors and the leading cause of cancer death in men. As is reported, prostate cancer comes in the second place after lung cancer in men (Labbé et al. 2018). In addition to age, race and family history of prostate cancer, prostate cancer susceptibility genes are also a key factor in the development of prostate cancer (Sathianathen et al. 2018). Relevant studies have confirmed that some DNA repair genes, including CHEK2, PALB2, BRIP1 and NBS1, may lead to a higher risk of prostate cancer. The risk assessment of prostate cancer usually combines the patient's age, clinical tumor stage, serum 
PSA concentration, and Gleason score for a comprehensive analysis (Barata et al. 2019). Among them, EDCs play a key role in the occurrence and development of prostate cancer (Hu et al. 2012). Androgen receptor (AR) is very important for prostate development and tumorigenic growth. A large amount of evidence demonstrated that human exposure to EDCs can change the function of hormones, and finally promote the occurrence and development of prostate cancer (Litwin et al. 2017). As an endocrine disruptor that interact with hormone, BPA can directly interact with both ER- $a$ and ER- $\beta$ and may enhance prostate cancer growth (Shafei et al. 2018). Besides, patients with prostate cancer showed higher levels of BPA in their urine, which suggests that urine BPA levels may have prognostic value for prostate cancer (Hess-Wilson. 2005). As an alternative for BPA, BPS has been increasingly detected in consumer products, foodstuffs, water, sediment and sludge in recent years (Fossati et al. 2016). Studies have reported the occurrence of BPS in human urine, among 315 urine samples collected from the United States, BPS was found in $81 \%$ of the total samples, and the average concentration of BPS was 0.17 $\mathrm{ng} / \mathrm{ml}$, which indicated that BPS may have toxic effects on the genitourinary system (Eladak et al. 2014).

Although the epidemiological literature on the toxicity of BPS are growing rapidly, however, its quantity and quality are still limited. Many studies are essentially cross-sectional studies, which makes results difficult to verify. The Comparative Toxicogenomics Database (CTD) is a literature-based and manually curated public resource and give us advance understanding regarding the effect of environmental exposure on human health. Besides, two tumor databases are also applied for further analysis. The Cancer Genome Atlas (TCGA), as a landmark cancer genomics program, molecularly characterized over 20,000 primary cancer and matched normal samples spanning 33 cancer types. GEO is a public functional genomics data repository supporting MIAME-compliant data submissions and provide arraybased and sequence-based data. The development of prostate cancer involves the damages to the normal prostate transcription network. Here, we evaluate the key genes that associated with prostate cancer. Besides, the interaction of toxins with their interaction genes helps understand the multiple pharmacology of bisphenols and their effects on biological networks. TCGA and GEO database were applied to explored the possible mechanism underlying the association between prostate cancer and bisphenols-exposure.

\section{Method}

\section{Datasets downloaded}

The data of interactive genes that associated with bisphenols were obtained from the CTD database (http://ctdbase.org/) in July 2021. Data on 8 bisphenols (Bisphenol A, Bisphenol AF, Bisphenol B, Bisphenol C, Bisphenol E, Bisphenol F, Bisphenol S, Bisphenol Z), as well as their gene, protein, and disease interactions, were downloaded. On the basis of 2D structure of bisphenols, they have similarities in structure. Two phenol groups are connected to a carbon atom at the 4'position. (Fig. 1)

\section{GO and KEGG pathway enrichment analysis}


In order to explore the potential biological processes (BPs), cellular component (CC) and molecular functions (MFs) of the interactive genes associated with bisphenols, Gene Ontology (GO) and Kyoto Encyclopedia of Genes and Genomes (KEGG) functional annotation analyses were applied. The "clusterProfiler" and "ggplot2" packages in R were used to perform GO and KEGG functional annotation pathway enrichment analysis. $P<0.05$ was selected as the threshold in the analysis.

\section{Protein-protein interaction network}

In order to further explore the relationship between interactive genes and protein, the protein-protein interaction (PPI) network of interaction genes associated with bisphenol was conducted by using STRING (https://www.string-db.org/). We next counted the nodes associated with each interaction gene. Then, Cytoscape 3.7.2 was applied to analyze the PPI network.

\section{Target prediction}

The structures of bisphenols (Bisphenol A, Bisphenol AF, Bisphenol B, Bisphenol C, Bisphenol E, Bisphenol F, Bisphenol S, Bisphenol Z) were obtained from PubChem (https://pubch em.ncbi.nlm.nih.gov/). Then, we the structure of the bisphenols was transferred into SDF format. PharmMapper database (http://lilab.ecust.edu.cn/pharmmapper/) is an updated integrated pharmacophore matching platform with statistial method for potential target identification. The Images of the 3D bisphenols metabolites structure are uploaded to the Pharmmapper database to determine the prediction targets related to bisphenols metabolites. Finally, in order to increase the credibility of the results, all the predicted targets in bisphenols metabolites were uploaded to the UniProt database (https://www.uniprot.org/) for screening.

\section{Processing of data sets and screening of differentially expressed genes}

The transcription profile data sets were obtained from TCGA (https://portal.gdc.cancer.gov) and GEO (http://www.ncbi.nlm.nih.gov/ geo/) database, which included 1597 tumor tissues. Then, "limma" package in R were applied for further analysis (limma, https://bioconductor.org/packages/limma/).

\section{Identification of prognosis based on interactive genes}

In order to explore the key genes associated with prostate cancer and bisphenol. Kaplan-Meier survival curves was used to compare the prognosis difference between two groups. We downloaded the data from the TCGA-PC datasets. $P<0.05$ was defined as a significant difference.

\section{Validation of the interactive genes involved in prostate cancer and bisphenol}

Receiver operating characteristic (ROC) curve was used to assess the stability of the prognosis model. The area under roc curve (AUC) value $>0.6$ was considered to have good predictive capabilities.

\section{Gene set variation analysis of key genes}


Gene set variation analysis of key genes was conducted to compare the difference of biological pathways between two groups. The reference gene set was "Hallmark.7.4.symbol.gmt".

\section{Cell Counting Kit-8 Assay}

CCK8 assay were performed using a CCK8 kit (Dojindo, Shanghai, China). Briefly, cells were plated into 96-well plates with 2000 cell per well and added $10 \mu \mathrm{L}$ CCK8. Cells were maintained for $1.5 \mathrm{~h}$ in a humidified incubator at $37^{\circ} \mathrm{C}$ with $5 \% \mathrm{CO} 2$. The absorbance at $450 \mathrm{~nm}$ was used to express the cell proliferation ability.

\section{RNA isolation and quantitative real-time PCR}

The total RNA was isolated from cell lines using TRIzol reagent (Invitrogen, CA, USA) according to the manufacturer's protocol. The cDNA was synthesized with PrimeScript ${ }^{\mathrm{TM}} \mathrm{RT}$ reagent Kit (Takara). The purity and concentration of RNAs were assessed by Nanodrop ND-2000 spectrophotometer (Thermo Scientific, EUA). Quantitative real-time PCR (qRT-PCR) was conductedusing AceQ $®$ qPCR SYBR Green Master Mix (vazyme) and according to the manufacturer's instructions with the Roche LightCycler 480 (Applied Biosystems, CA, USA). GAPDH was used as internal control and each sample was repeated four times. The fold change in the expression of genes was calculated with the formula 2- $\triangle \triangle C T$. The primer sequence used was as follows: COL1A1, forward primer, 5'-GAGGGCCAAGACGAAGACATC-3', reverse primer, 5'-CAGATCACGTCATCGCACAAC-3'; COL1A2, forward primer, 5'-GTTGCTGCTTGCAGTAACCTT-3', reverse primer, 5'-AGGGCCAAGTCCAACTCCTT-3'.

\section{Cell migration assays}

Cell migration was determined by the wound healing assay and the transwell assay. (1) The woundhealing assay. $4 \times 10^{5}$ cells were seeded on 12 well culture plates in DMEM supplemented with $10 \%$ FBS for 24 hours. After the cells reached to about $80-90 \%$ confluence in a monolayer, a pipette tip was used to make a straight scratch line in the cell monolayer. The cells were incubated for indicated times and treated with BPS at the concentration of $10^{-8} \mathrm{M}$. (2) Transwell assay. Transwell cell with a pore size of 8.0um was added to a 24 -well plate to form upper and lower cells. Next, 600 ul of medium containing $10 \%$ FBS were added in the lower chamber, 200 portions of serum-free conditioned medium containing $2 * 104$ cells and BPS with different concentration gradients were added in the upper chamber. After 24 hours, wipe the upper chamber cells with a cotton swab, fix the invading cells, and count by crystal violet staining.

\section{Statistical analysis}

All the analysis were conducted in R software v4.0.0, SPSS v13.0 and GraphPad Prism 8. P-value was two-side and $<0.05$ was regarded as statistically significant. Student T-test were used to compare the difference between the two groups.

\section{Results}




\section{Enrichment analysis of interactive genes affected by bisphenols}

On the basis of CTD database, we obtained interactive genes of Bisphenol A, Bisphenol AF, Bisphenol B, Bisphenol C, Bisphenol E, Bisphenol F, Bisphenol S and Bisphenol Z. After summarizing the data, a total of 24041 interactive genes were obtained. Among the genes with interactions, we selected interactive genes with more than 15 interaction counts for further analysis, includes some genes with strong interaction counts INS (3934 interaction counts), ESR1 (771 interaction counts), AR (263 interaction counts), ESR2 (229 interaction counts), MAPK1 (165 interaction counts), MAPK3 (164 interaction counts), LIF (128 interaction counts), PPARG (115 interaction counts), Then we select the interactive genes that demonstrate more than 15 interactions for GO and KEGG analysis to figure out the BPs, CC and MFs.

In terms of BPs (Fig. 2A), response to estradiol, response to drug, response to peptide hormone, regulation of lipid metabolic process and gland development were the most enrichment results. Estradiol is a key hormone which plays an important role in hypothalamic-pituitary-testicular axis regulation, reproductive function and body composition (Luine et al. 2014). Estradiol, ERa, ER $\beta$ can limit the growth and spread of prostate cancer cells and prevent prostate cancer from progressing towards castration-resistance forms (Russell et al. 2019). Lipid metabolism may promote the growth of prostate cancer and the resistance to endocrine therapy (Stoykova et al. 2019).

The GO enrichment analysis of interactive genes demonstrated that CC were most associated with vesicle lumen, membrane raft, membrane microdomain, membrane region, secretory granule lumen and cytoplasmic vesicle lumen. Membrane raft is the microdomain which is enriched in cholesterol and sphingomyelin (Sonnino et al. 2013). Prostate cancer contains high levels of cholesterol, which means that larger rafts can be formed in these cell membranes, stimulating signaling pathways to promote tumor growth and progression (Hryniewicz-Jankowska et al. 2019). (Fig. 2B)

For MF, the most enriched terms included nuclear receptor activity, ligand-activated transcription factor activity, steroid binding, DNA-binding transcription factor binding, RNA polymerase II-specific DNA-binding transcription factor binding and receptor ligand activity. As a ligand-activated transcription factor, the androgen receptor (AR) drives prostate cancer and plays an important role in the mainstay treatment for men with metastatic disease (Gabriely et al. 2017). (Fig. 2C)

We finally conducted KEGG enrichment analysis by identifying 219 interactive genes to figure out the most relative diseases associated with bisphenol. After screening the first 20 pathways related to bisphenol, the most enriched pathway was prostate cancer. Besides, bisphenol is also closely related to hepatitis B, reproductive system development and endocrine resistance. Moreover, KEGG pathway enrichment analysis demonstrated that bladder cancer may also associated with bisphenol. (Fig. 2D)

\section{PPI network of key genes associated with prostate cancer and bisphenol}


The PPI network was constructed by String (https://www.string-db.org/). Next, the cytoscape 3.8.2 was applied to analyze PPI network and further explore the roles of interactive genes involved in bisphenol. The minimum required interaction score was set as 0.9 and the degree value is correlated with node size. After comprehensive analysis of interactive genes, a total of 219 nodes and 2103 edges were identified. Our results revealed that the interactive genes demonstrated strong protein-protein interaction. Some interactive genes, including JUN, CREBBP, AKT1, MAPK1, STAT3, TP53 NCOA1, MAPK3, ESR1, SRC, PPARG, VEGFA, NCOA2 showed more than 30 degrees. (Fig. 3)

\section{Target prediction for bisphenol}

The 2D chemical structure of bisphenols were obtained from PubChem. With the help of Pharmmapper database, a total of 796 predicted targets of bisphenols were found. In order to figure out the potential relationship between prostate cancer and bisphenols, we used the KEGG analysis to figure out its underly pathway. We found that AR was considered as key targets of bisphenols, which also play a key role in the progression of prostate cancer. (Fig. 4)

\section{Screening of differential expression genes DEGs}

RNA-seq data from the GEO database and TCGA database were applied to compare the DEGs between high-risk group and low-risk group based on gleason score. After data download and preprocessing by using the Perl script, we obtained a total of 213 patients with low-gleason score and 209 patients with high-gleason score. The results demonstrated that 57 downregulated and 22 upregulated genes in the prostate of prostate cancer patients with high-gleason score compared with those in the prostate of prostate cancer patients with low-gleason score. (Fig. 5A) The standard was $\mathrm{P}<0.05$ and absolute $\log 2 \mathrm{FC}>0.5$. After screening the prostate cancer-associated genes, venn diagram was conducted to explored the genes associated with prostate cancer and bisphenol. COL1A1 and COL1A2 were defined as bisphenol-exposure genes associated with prostate cancer. (Fig. 5B)

\section{Function and interaction analysis of interactive genes}

In order to figure out the impact of bisphenol-exposure genes associated with prostate cancer on prostate cancer patients, transcriptome data and clinical data of prostate cancer patients were downloaded from TCGA-PRAD. Disease-free survival (DFS) was applied for the measurement of survival analysis. The results showed that prostate cancer patients with high expression of COL1A1 and COL1A2 were related to the poor DFS. (Fig. 6A,B) On the basis of survival analysis of the bisphenol-exposure genes associated with prostate cancer, COL1A1 and COL1A2 showed strong function of the prediction in the prognosis in prostate cancer. Time-dependent ROC curves showed AUC of COL1A1 and COL1A2 are 0.685 and 0.625 respectively. Based on the survival analysis and AUC values, COL1A1 and COLIA2 showed strong value in the prediction of prostate cancer. (Fig. 6C) 


\section{Gene set variation analysis (GSVA) between low-expression and high-expression groups of COL1A1 and COLIA2}

In the previous work, survival analysis and ROC curves have conducted to prove the function of COL1A1 and COL1A2 in prostate cancer and bisphenol-exposure. In order to further explore the potential function of COL1A1 and COL1A2, GSVA of hallmark gene sets were conducted by "limma","stringr" packages. The results demonstrated that collagen trimer, cell cycle and transporter activity were highly activated.

(Fig. 7A,B)

\section{Cell proliferation and invasion of prostate cells with BPS- treated}

The CCK8 assay was applied to figure out the cell proliferation ofboth PC-3 and LNCap cells with the BPS-treated $\left(10^{-8} \mathrm{M}\right)$. After treated with BPS for 72 hours, we found that BPS significantly increased the cell proliferation ability in both PC-3 and LNCap cells compared with the control group $(p<0.05)$ (Fig. 8A$B$ ). The results of transwell assay showed that the BPS could promote the cell invasion of PC-3 and LNCap cells (Fig. 8C-D). Wound-healing assay demonstrated that BPS significantly promoted the migratory capacities of in both PC-3 and LNCap cells(Fig. 8E-F). Besides, COL1A1 and COL1A2 in the PC3 and LNCap cells treated with $10^{-8} \mathrm{M}$ BPS were significantly upregulated compared with control group (Fig. 8G-H).

\section{Discussion}

As an industrial component with good strength and hardness, thermal stability and resistance to acids and oils, bisphenol has been widely used in industrial production, including materials for the production of phenolic resin, polyacrylate, polyester, epoxy resin and polycarbonate plastics (Mao et al. 2020). However, due to its toxicity and estrogen-like effects, manufacturers start to remove BPA from their products and look for alternative chemicals (Huang et al. 2020). As the two mostly used substitute for BPA, BPS and BPF has been detected in many daily products, including personal care products, paper products and food. BPS and BPF are structural analogues of BPA, therefore, their effects in the physiological system may be similar (Xie et al. 2020). The rodent studies reported that BPS or BPF exposure increased female to male sex ratio, reduced the testosterone in male rodents, damaged the sperm parameters (Thoene et al. 2020). Prostate cancer demonstrated a higher prevalence in western and immigrant population, which implies lifestyle and environmental may be the risk factors (Komura et al. 2018). As a tumor with the high mortality rate in men, it has long been known that prostate cancer is closely related to androgen receptor (Chang et al. 2014). Androgen-receptor signaling is altered in castration-resistant prostate cancer (Liu et al. 2018). EDCs with estrogenic activity is associated with the increased risk of prostate cancer. Cancer stem cells are potential factors for the occurrence and progression of cancer. EDCs with estrogenic activity may influence the occurrence and progression of 
prostate cancer by reprogramming and transforming prostate stem and early progenitor cells (Zhang et al. 2018).

In recent years, due to the rapid development of network databases, many disciplines have begun to use databases for forecasting work. As an emerging research tool, analysis using the database can provide us with the correct direction for our subsequent experiments. In this work, in order to figure out the potential relationship between prostate cancer and bisphenol, bioinformatics approach was applied by silico analysis. On the basis of CTD and TCGA databases, we successfully discovered the potential tumor pathway of bisphenol. KEGG enrichment analysis demonstrated that many types of tumors, including prostate cancer, bladder cancer, colorectal cancer and thyroid cancer were highly enriched, which prostate cancer is the most enriched cancer. In order to discover the most related genes between prostate cancer and bisphenol, TCGA and GEO database were applied. Further investigations suggested that several interactive genes were strongly correlated with the progression of prostate cancer. We believe that these two interactive genes (COL1A1 and COL1A2) have predictive effects and may play an important role in promoting the development of prostate cancer induced by bisphenols. By comprehensive analyses of public databases, our work successfully evaluated the associations between bisphenols in the CTD database with the molecular markers of prostate cancer. Our study results provided novel clues for studies on the pathogenetic and risk factors of prostate cancer.

In previous studies, COL1A1 and COL1A2 was considered to be a downstream effector of cytoglobin associated with many common malignancies (Ma et al. 2019). In addition, the increase of COL1A1 and COL1A2 expression in colon cancer is significantly related to serous membrane infiltration, lymphatic metastasis and hematogenous metastasis. COL1A1 and COL1A2 can be used as a candidate diagnostic biomarker for colon cancer. Our study found that COL1A1 and COL1A2 can promote the progression of prostate cancer. Our data demonstrated that BPS significantly increase the cell proliferation ability and promote cell invasion capacity. COL1A1 and COL1A2 in the PC-3 and LNCap cells were significantly upregulated with BPS treated. Our research will help further understand the adverse effects of bisphenols and provide direction to future studies on bisphenols.

\section{Conclusion}

As a substitute for BPA, the application of BPS has been increasing over the decades. However, since BPS is considered to be highly toxic to mammals, BPS has attracted more and more attention. In this research, we explored the genes affected by BPS and its potential targets. Our results demonstrated that BPS was closely related to prostate cancer, Further studies proved COL1A1 and COL1A2 played an important role in predicting the prognosis and progression of prostate cancer. Besides, we verified that BPS can promote the proliferation and invasion of prostate cancer cell lines.

\section{Declarations}

\section{Ethical Approval}


Not applicable.

\section{Consent to Participate}

Not applicable.

\section{Consent to Publish}

The authors agree the publication in the Environmental science and pollution research.

\section{Authors Contributions}

$\mathrm{DL}, \mathrm{YL}$ and $\mathrm{KF}$ collected the data and performed the meta-analysis. DL,SL,SL and KF wrote the manuscript. All authors contributed to the article and approved the submitted version.

\section{Funding}

This work was supported by Science and Technology Development Fund of Shanghai Pudong New Area (Grant No. PKJ2018-Y34)

This work was supported by the Natural Science Foundation of China (Project No. 81802547)

\section{Competing Interests}

The authors declare that they have no known competing financial interests or personal relationships that could have appeared to influence the work reported in this paper.

\section{Availability of data and materials}

Data and materials are available for manuscript.

\section{References}

1. Barata PC, Sartor AO. Metastatic castration-sensitive prostate cancer: Abiraterone, docetaxel, or.... Cancer. 2019 Jun 1;125(11):1777-1788. doi: 10.1002/cncr.32039. Epub 2019 Apr 1. PMID: 30933324.

2. Chang AJ, Autio KA, Roach M 3rd, Scher HI. High-risk prostate cancer-classification and therapy. Nat Rev Clin Oncol. 2014 Jun;11(6):308-23. doi: 10.1038/nrclinonc.2014.68. Epub 2014 May 20. PMID: 24840073; PMCID: PMC4508854.

3. Eladak S, Grisin T, Moison D, Guerquin MJ, N'Tumba-Byn T, Pozzi-Gaudin S, Benachi A, Livera G, Rouiller-Fabre V, Habert R. A new chapter in the bisphenol A story: bisphenol $\mathrm{S}$ and bisphenol $\mathrm{F}$ are not safe alternatives to this compound. Fertil Steril. 2015 Jan;103(1):11-21. doi: 10.1016/j.fertnstert.2014.11.005. Epub 2014 Dec 2. PMID: 25475787. 
4. Fossati N, Willemse PM, Van den Broeck T, van den Bergh RCN, Yuan CY, Briers E, Bellmunt J, Bolla M, Cornford P, De Santis M, MacPepple E, Henry AM, Mason MD, Matveev VB, van der Poel HG, van der Kwast TH, Rouvière O, Schoots IG, Wiegel T, Lam TB, Mottet N, Joniau S. The Benefits and Harms of Different Extents of Lymph Node Dissection During Radical Prostatectomy for Prostate Cancer: A Systematic Review. Eur Urol. 2017 Jul;72(1):84-109. doi: 10.1016/j.eururo.2016.12.003. Epub 2017 Jan 24. PMID: 28126351.

5. Gabriely G, Wheeler MA, Takenaka MC, Quintana FJ. Role of AHR and HIF-1a in Glioblastoma Metabolism. Trends Endocrinol Metab. 2017 Jun;28(6):428-436. doi: 10.1016/j.tem.2017.02.009. Epub 2017 Mar 16. PMID: 28318896; PMCID: PMC5438779.

6. Giri VN, Knudsen KE, Kelly WK, Cheng HH, Cooney KA, Cookson MS, Dahut W, Weissman S, Soule HR, Petrylak DP, Dicker AP, AIDubayan SH, Toland AE, Pritchard CC, Pettaway CA, Daly MB, Mohler JL, Parsons JK, Carroll PR, Pilarski R, Blanco A, Woodson A, Rahm A, Taplin ME, Polascik TJ, Helfand BT, Hyatt C, Morgans AK, Feng F, Mullane M, Powers J, Concepcion R, Lin DW, Wender R, Mark JR, Costello A, Burnett AL, Sartor O, Isaacs WB, Xu J, Weitzel J, Andriole GL, Beltran H, Briganti A, Byrne L, Calvaresi A, Chandrasekar T, Chen DYT, Den RB, Dobi A, Crawford ED, Eastham J, Eggener S, Freedman ML, Garnick M, Gomella PT, Handley N, Hurwitz MD, Izes J, Karnes RJ, Lallas C, Languino L, Loeb S, Lopez AM, Loughlin KR, Lu-Yao G, Malkowicz SB, Mann M, Mille P, Miner MM, Morgan T, Moreno J, Mucci L, Myers RE, Nielsen SM, O'Neil B, Pinover W, Pinto P, Poage W, Raj GV, Rebbeck TR, Ryan C, Sandler H, Schiewer M, Scott EMD, Szymaniak B, Tester W, Trabulsi EJ, Vapiwala N, Yu EY, Zeigler-Johnson C, Gomella LG. Implementation of Germline Testing for Prostate Cancer: Philadelphia Prostate Cancer Consensus Conference 2019. J Clin Oncol. 2020 Aug 20;38(24):27982811. doi: 10.1200/JC0.20.00046. Epub 2020 Jun 9. PMID: 32516092; PMCID: PMC7430215.

7. Grozescu T, Popa F. Prostate cancer between prognosis and adequate/proper therapy. J Med Life. 2017 Jan-Mar;10(1):5-12. PMID: 28255369; PMCID: PMC5304372.

8. Hu WY, Shi GB, Hu DP, Nelles JL, Prins GS. Actions of estrogens and endocrine disrupting chemicals on human prostate stem/progenitor cells and prostate cancer risk. Mol Cell Endocrinol. 2012 May 6;354(1-2):63-73. doi: 10.1016/j.mce.2011.08.032. Epub 2011 Sep 5. PMID: 21914459; PMCID: PMC3249013.

9. Hess-Wilson JK, Knudsen KE. Endocrine disrupting compounds and prostate cancer. Cancer Lett. 2006 Sep 8;241(1):1-12. doi: 10.1016/j.canlet.2005.10.006. Epub 2005 Nov 17. PMID: 16298040.

10. Huang M, Liu S, Fu L, Jiang X, Yang M. Bisphenol A and its analogues bisphenol S, bisphenol $F$ and bisphenol AF induce oxidative stress and biomacromolecular damage in human granulosa KGN cells. Chemosphere. 2020 Aug;253:126707. doi: 10.1016/j.chemosphere.2020.126707. Epub 2020 Apr 9. PMID: 32289607.

11. Hryniewicz-Jankowska A, Augoff K, Sikorski AF. The role of cholesterol and cholesterol-driven membrane raft domains in prostate cancer. Exp Biol Med (Maywood). 2019 Oct;244(13):1053-1061. doi: 10.1177/1535370219870771. PMID: 31573840; PMCID: PMC6775568.

12. Komura K, Sweeney CJ, Inamoto T, Ibuki N, Azuma H, Kantoff PW. Current treatment strategies for advanced prostate cancer. Int J Urol. 2018 Mar;25(3):220-231. doi: 10.1111/iju.13512. Epub 2017 
Dec 20. PMID: 29266472; PMCID: PMC6053280.

13. Komura K, Sweeney CJ, Inamoto T, Ibuki N, Azuma H, Kantoff PW. Current treatment strategies for advanced prostate cancer. Int J Urol. 2018 Mar;25(3):220-231. doi: 10.1111/iju.13512. Epub 2017 Dec 20. PMID: 29266472; PMCID: PMC6053280.

14. Liu J, Shen JX, Wu HT, Li XL, Wen XF, Du CW, Zhang GJ. Collagen 1A1 (COL1A1) promotes metastasis of breast cancer and is a potential therapeutic target. Discov Med. 2018 May;25(139):211-223. PMID: 29906404.

15. Labbé DP, Brown M. Transcriptional Regulation in Prostate Cancer. Cold Spring Harb Perspect Med. 2018 Nov 1;8(11):a030437. doi: 10.1101/cshperspect.a030437. PMID: 29530947; PMCID: PMC6211375.

16. Legeay S, Faure S. Is bisphenol A an environmental obesogen? Fundam Clin Pharmacol. 2017 Dec;31(6):594-609. doi: 10.1111/fcp.12300. Epub 2017 Jul 7. PMID: 28622415.

17. Litwin MS, Tan HJ. The Diagnosis and Treatment of Prostate Cancer: A Review. JAMA. 2017 Jun 27;317(24):2532-2542. doi: 10.1001/jama.2017.7248. PMID: 28655021.

18. Murillo-Garzón V, Kypta R. WNT signalling in prostate cancer. Nat Rev Urol. 2017 Nov;14(11):683-696. doi: 10.1038/nrurol.2017.144. Epub 2017 Sep 12. PMID: 28895566.

19. Ma Y, Liu H, Wu J, Yuan L, Wang Y, Du X, Wang R, Marwa PW, Petlulu P, Chen X, Zhang H. The adverse health effects of bisphenol A and related toxicity mechanisms. Environ Res. 2019 Sep;176:108575. doi: 10.1016/j.envres.2019.108575. Epub 2019 Jul 3. PMID: 31299621.

20. Murata M, Kang JH. Bisphenol A (BPA) and cell signaling pathways. Biotechnol Adv. 2018 JanFeb;36(1):311-327. doi: 10.1016/j.biotechadv.2017.12.002. Epub 2017 Dec 8. PMID: 29229539.

21. Mao J, Jain A, Denslow ND, Nouri MZ, Chen S, Wang T, Zhu N, Koh J, Sarma SJ, Sumner BW, Lei Z, Sumner LW, Bivens NJ, Roberts RM, Tuteja G, Rosenfeld CS. Bisphenol A and bisphenol S disruptions of the mouse placenta and potential effects on the placenta-brain axis. Proc Natl Acad Sci U S A. 2020 Mar 3;117(9):4642-4652. doi: 10.1073/pnas.1919563117. Epub 2020 Feb 18. PMID: 32071231; PMCID: PMC7060676.

22. Ma HP, Chang HL, Bamodu OA, Yadav VK, Huang TY, Wu ATH, Yeh CT, Tsai SH, Lee WH. Collagen 1A1 (COL1A1) Is a Reliable Biomarker and Putative Therapeutic Target for Hepatocellular Carcinogenesis and Metastasis. Cancers (Basel). 2019 Jun 7;11(6):786. doi: 10.3390/cancers11060786. PMID: 31181620; PMCID: PMC6627889.

23. Rochester JR, Bolden AL. Bisphenol S and F: A Systematic Review and Comparison of the Hormonal Activity of Bisphenol A Substitutes. Environ Health Perspect. 2015 Jul;123(7):643-50. doi: 10.1289/ehp.1408989. Epub 2015 Mar 16. PMID: 25775505; PMCID: PMC4492270.

24. Sathianathen NJ, Konety BR, Crook J, Saad F, Lawrentschuk N. Landmarks in prostate cancer. Nat Rev Urol. 2018 Oct;15(10):627-642. doi: 10.1038/s41585-018-0060-7. PMID: 30065357.

25. Shafei A, Ramzy MM, Hegazy Al, Husseny AK, El-Hadary UG, Taha MM, Mosa AA. The molecular mechanisms of action of the endocrine disrupting chemical bisphenol $A$ in the development of 
cancer. Gene. 2018 Mar 20;647:235-243. doi: 10.1016/j.gene.2018.01.016. Epub 2018 Jan 6. PMID: 29317319.

26. Eladak S, Grisin T, Moison D, Guerquin MJ, N'Tumba-Byn T, Pozzi-Gaudin S, Benachi A, Livera G, Rouiller-Fabre V, Habert R. A new chapter in the bisphenol A story: bisphenol $\mathrm{S}$ and bisphenol $\mathrm{F}$ are not safe alternatives to this compound. Fertil Steril. 2015 Jan;103(1):11-21. doi: 10.1016/j.fertnstert.2014.11.005. Epub 2014 Dec 2. PMID: 25475787.

27. Luine VN. Estradiol and cognitive function: past, present and future. Horm Behav. 2014 Sep;66(4):602-18. doi: 10.1016/j.yhbeh.2014.08.011. Epub 2014 Sep 7. PMID: 25205317; PMCID: PMC4318702.

28. Russell N, Grossmann M. MECHANISMS IN ENDOCRINOLOGY: Estradiol as a male hormone. Eur J Endocrinol. 2019 Jul;181(1):R23-R43. doi: 10.1530/EJE-18-1000. PMID: 31096185.

29. Stoykova GE, Schlaepfer IR. Lipid Metabolism and Endocrine Resistance in Prostate Cancer, and New Opportunities for Therapy. Int J Mol Sci. 2019 May 28;20(11):2626. doi: 10.3390/ijms20112626. PMID: 31142021; PMCID: PMC6600138.

30. Sonnino S, Prinetti A. Membrane domains and the "lipid raft" concept. Curr Med Chem. 2013;20(1):421. PMID: 23150999.

31. Thoene M, Dzika E, Gonkowski S, Wojtkiewicz J. Bisphenol S in Food Causes Hormonal and Obesogenic Effects Comparable to or Worse than Bisphenol A: A Literature Review. Nutrients. 2020 Feb 19;12(2):532. doi: 10.3390/nu12020532. PMID: 32092919; PMCID: PMC7071457.

32. Xie C, Ge M, Jin J, Xu H, Mao L, Geng S, Wu J, Zhu J, Li X, Zhong C. Mechanism investigation on Bisphenol S-induced oxidative stress and inflammation in murine RAW264.7 cells: The role of NLRP3 inflammasome, TLR4, Nrf2 and MAPK. J Hazard Mater. 2020 Jul 15;394:122549. doi: 10.1016/j.jhazmat.2020.122549. Epub 2020 Apr 10. PMID: 32283380.

33. Zhang Z, Wang Y, Zhang J, Zhong J, Yang R. COL1A1 promotes metastasis in colorectal cancer by regulating the WNT/PCP pathway. Mol Med Rep. 2018 Apr;17(4):5037-5042. doi: 10.3892/mmr.2018.8533. Epub 2018 Feb 1. PMID: 29393423; PMCID: PMC5865965.

\section{Figures}


A

B
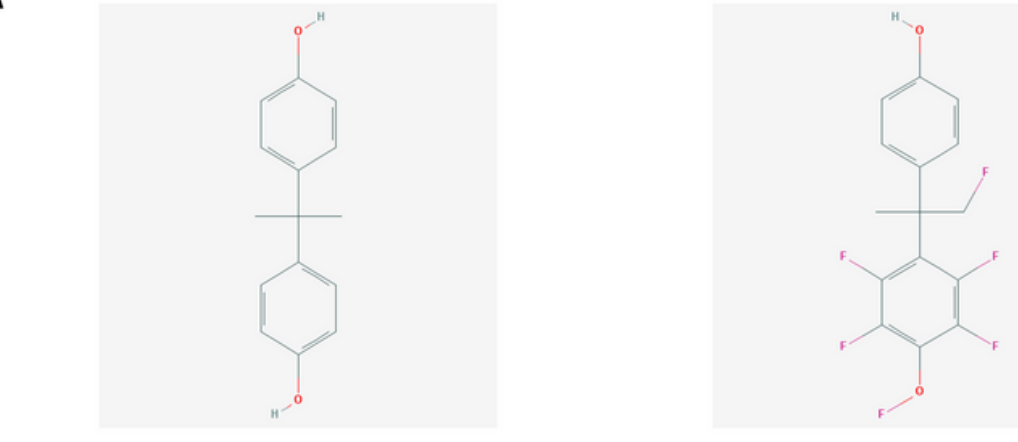

C

E

$\mathrm{F}$

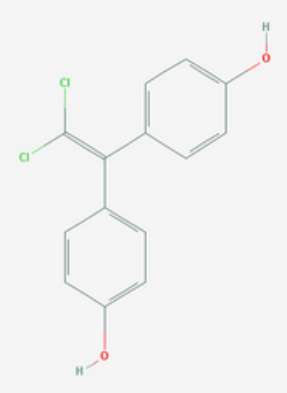

G

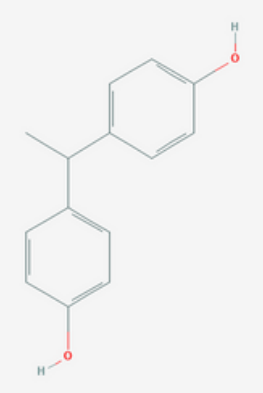

$\mathrm{H}$
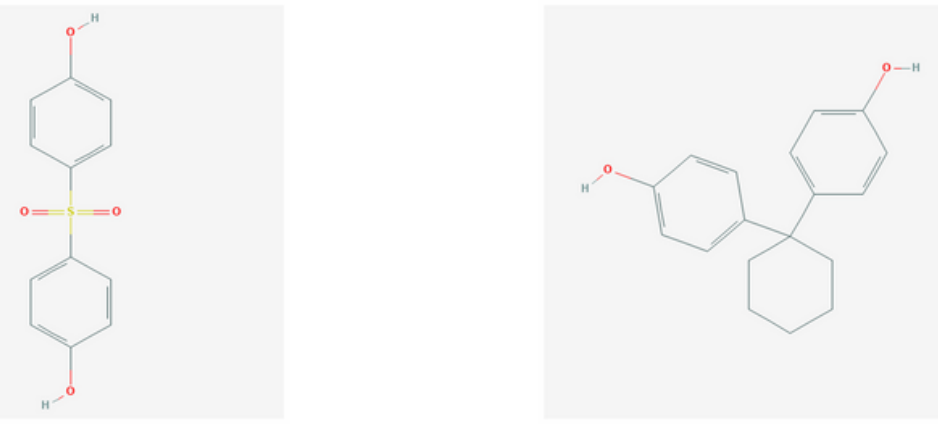

Figure 1

2D chemical structure of OPFRs and OPFRs metabolites. 
A
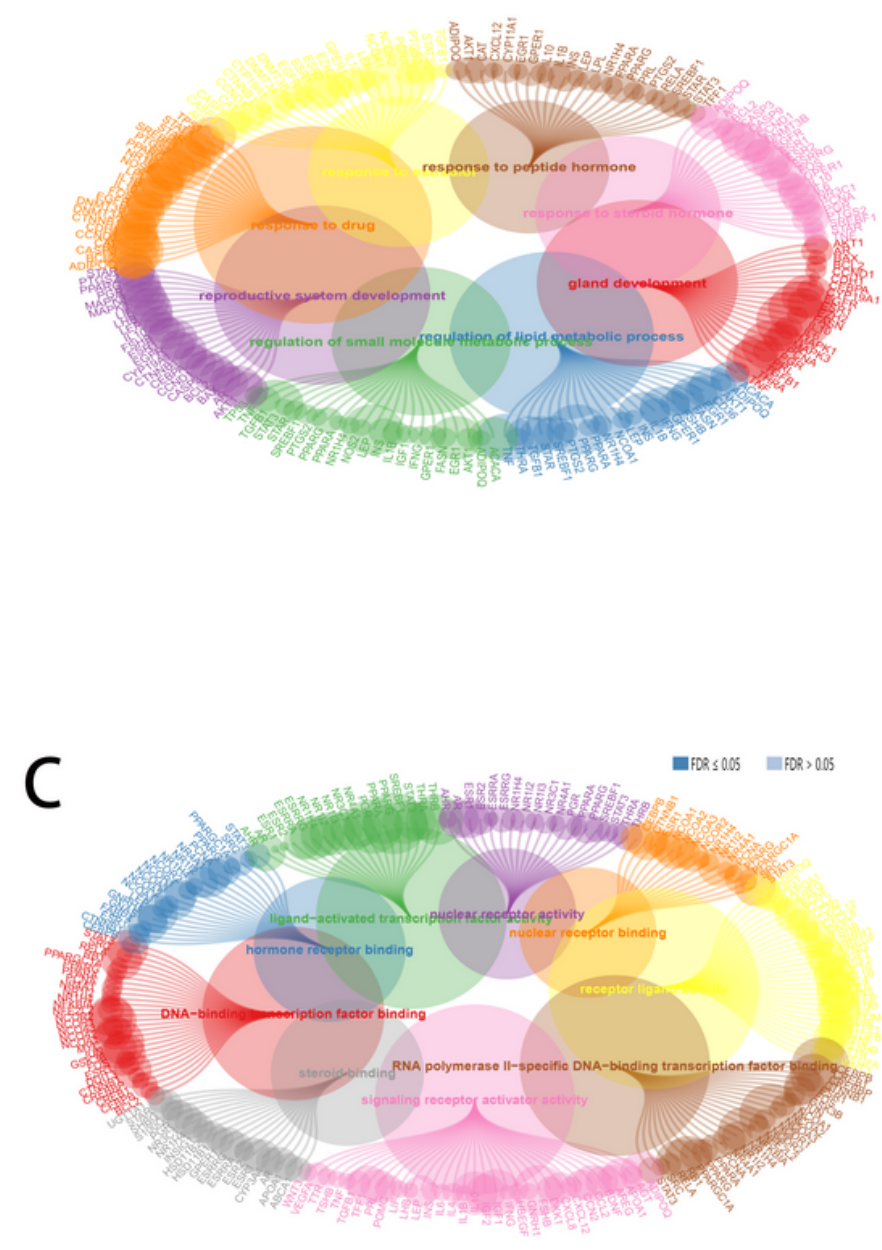

B

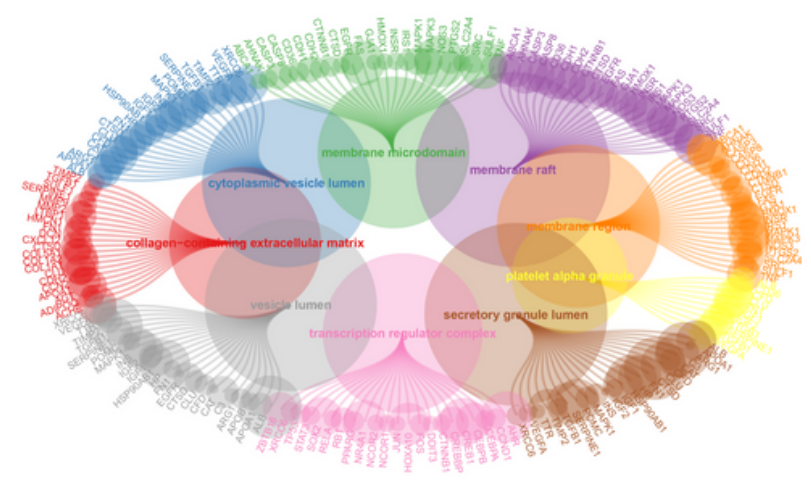

D

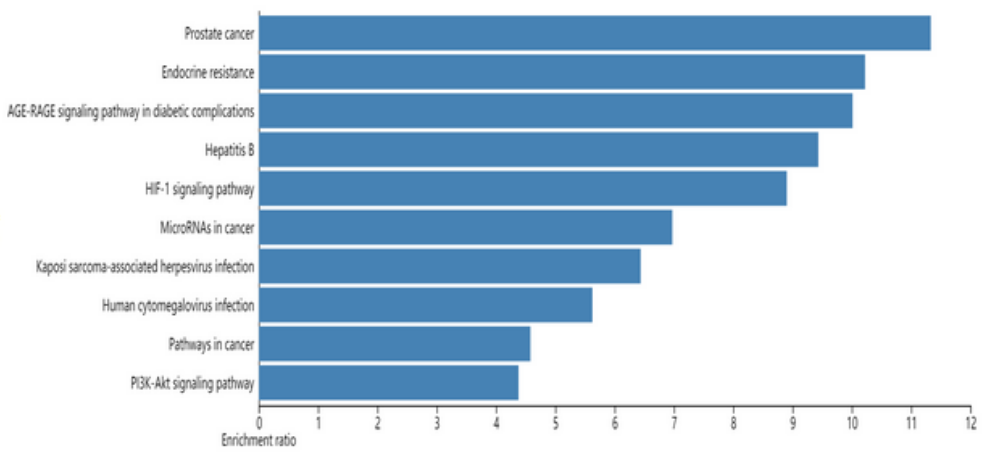

\section{Figure 2}

Circle plots were used to explore GO and KEGG enrichment pathways among differential expression genes. 


\section{A}
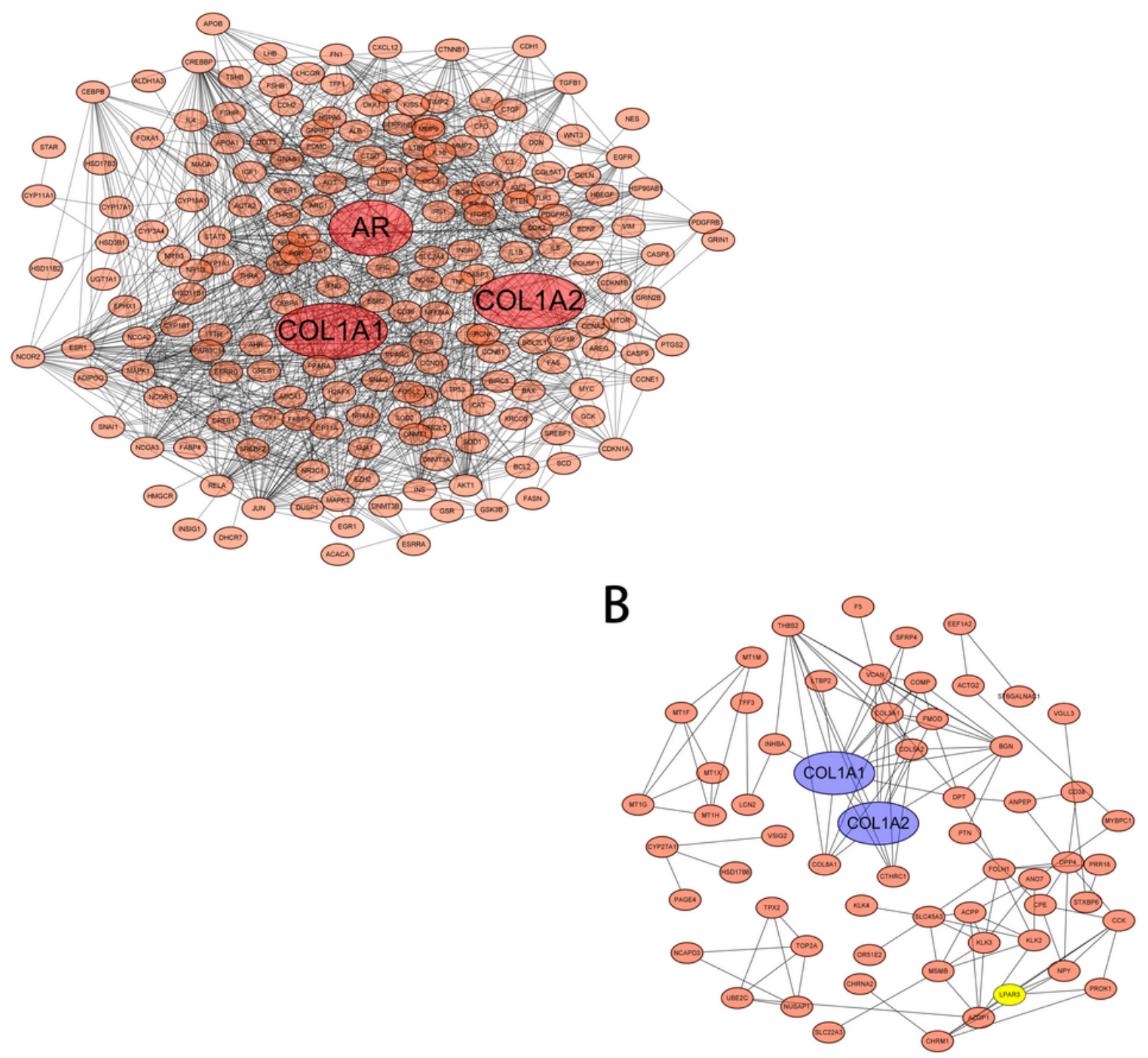

Figure 3

Construction of the PPI network and hub genes were identified. 
A

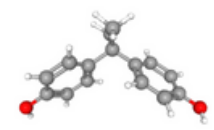

D

F

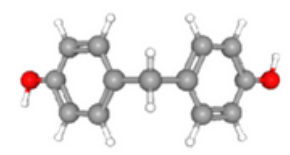

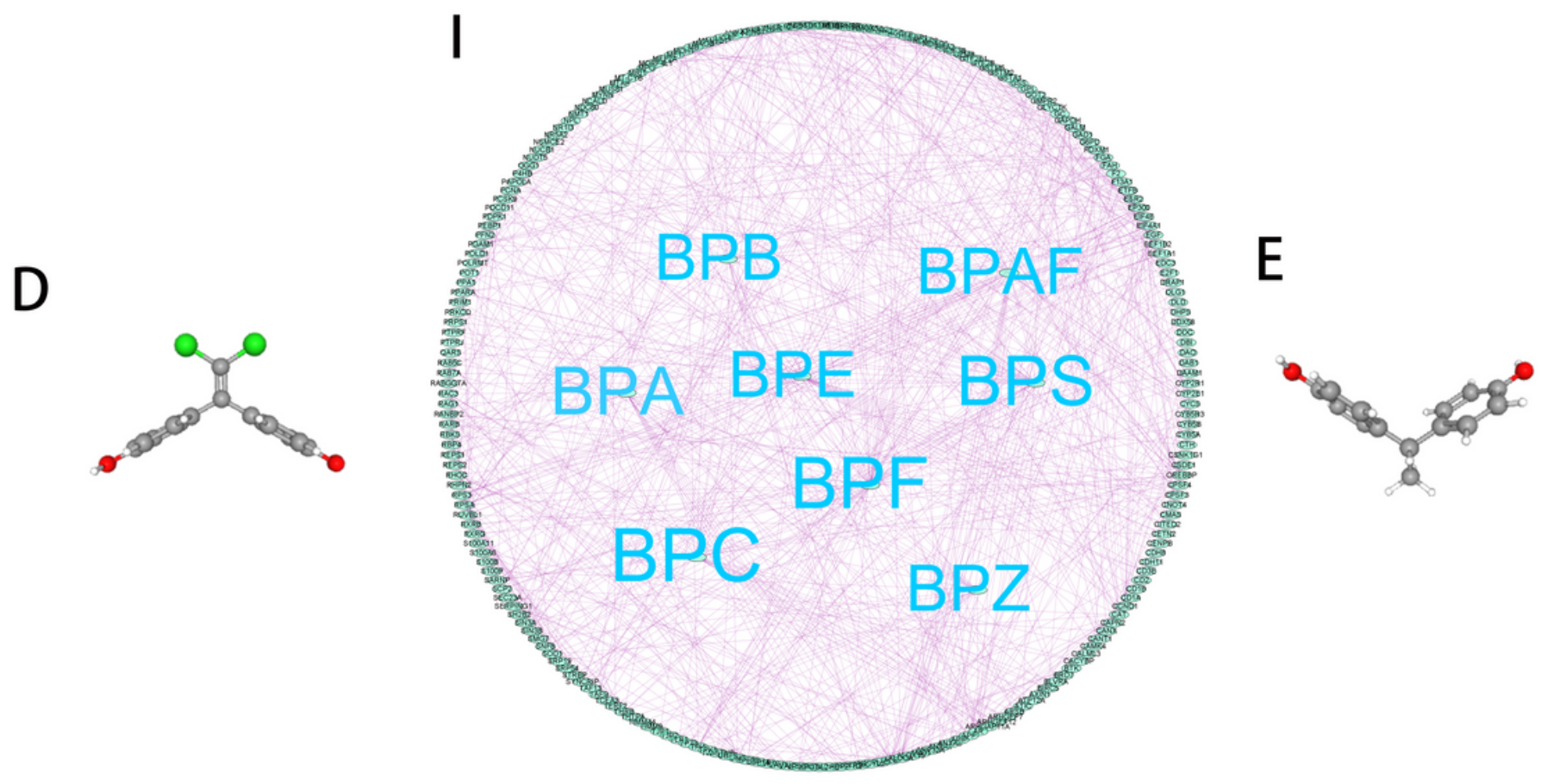

B

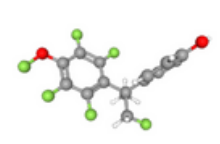

C

G

$\mathrm{H}$

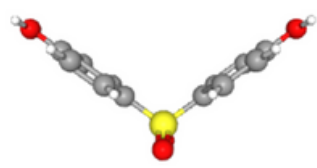

Figure 4

Targets analysis for bisphenols. 
A

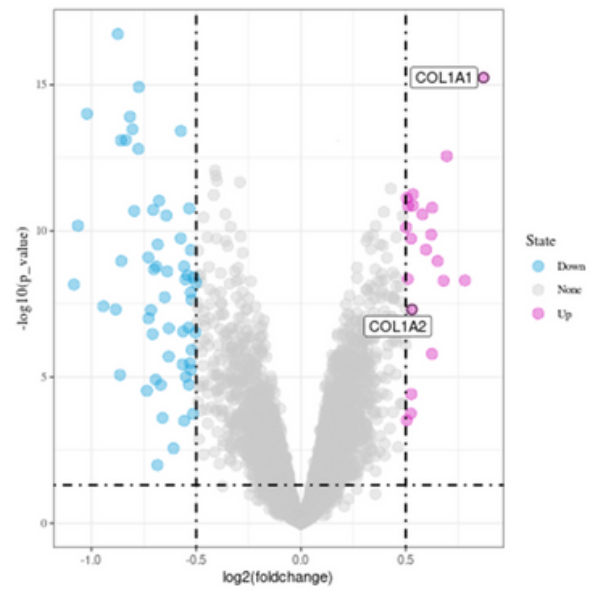

B

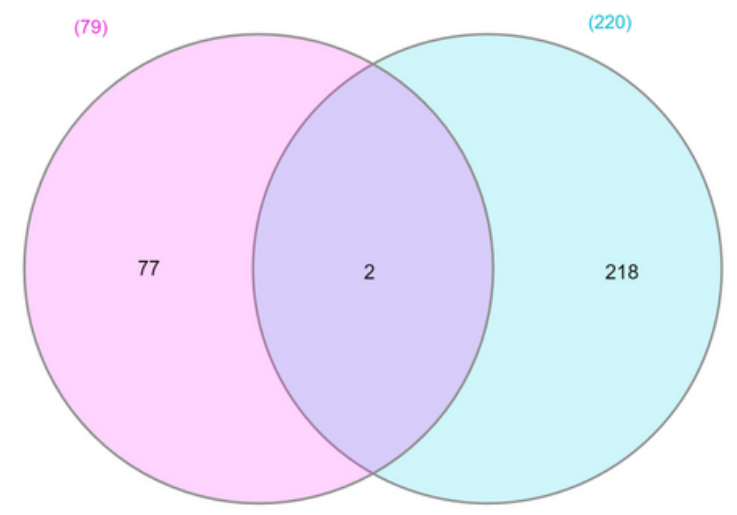

Figure 5

(A) Differentially expression genes in prostate cancer tissue samples. The differential expression genes were shown in the -log (P-value) vs. log (Fold-Change) plot. (B) Venn diagram demonstrated the relationship between prostate cancer and bisphenols. 
A

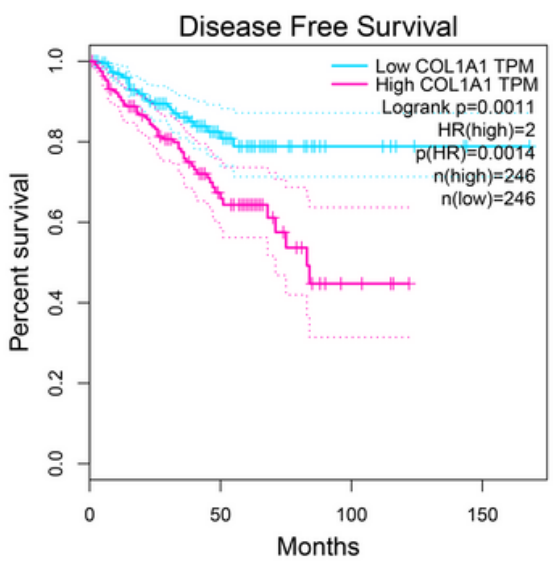

B

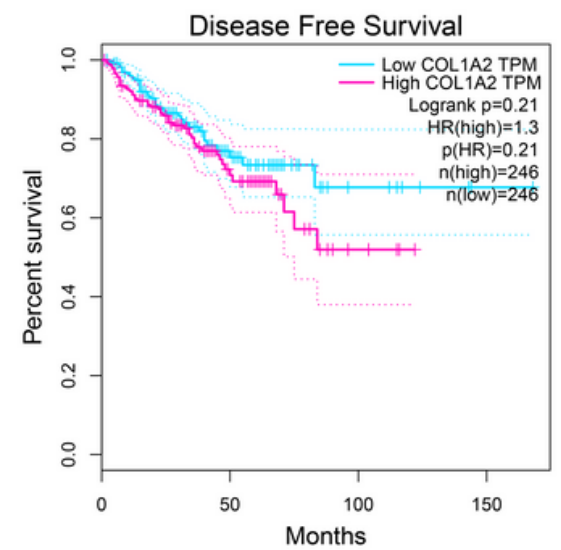

C

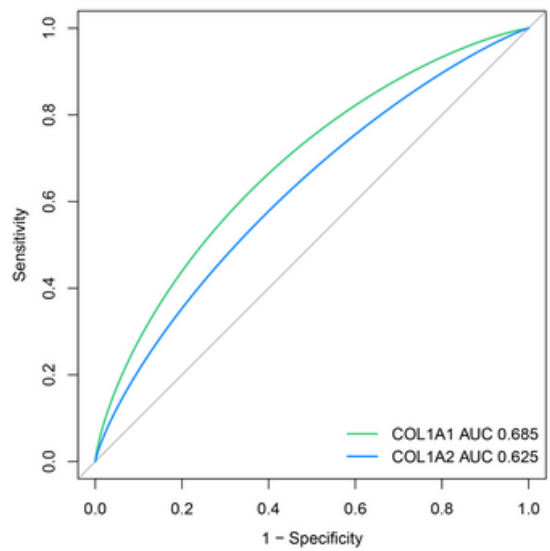

Figure 6

(A) Kaplan-Meier survival analysis for COL1A1 in TCGA-PRAD dataset. (B) Kaplan-Meier survival analysis for COL1A2 in TCGA-PRAD dataset. (C) ROC subgroup analyses showed that COL1A1 and COL1A2 expression might be a potential prognostic indicator with the prostate cancer. 
A

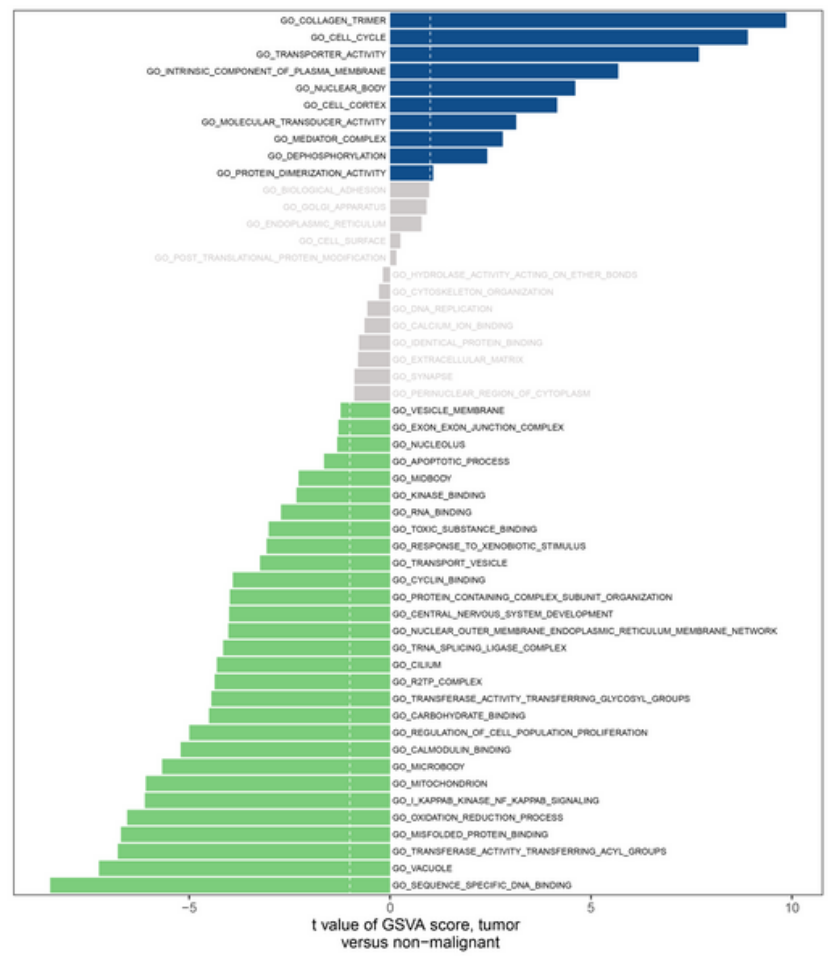

B

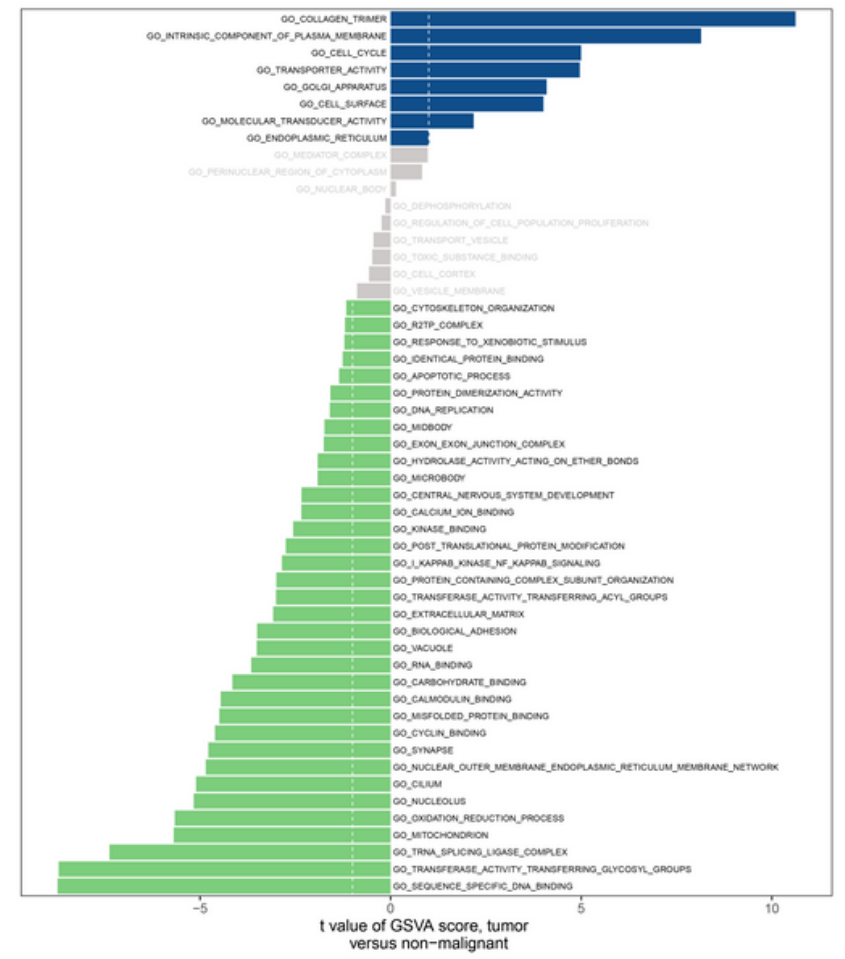

\section{Figure 7}

GSVA analysis in human prostate cancer tissue samples. (A): GSVA analysis between COL1A1 lowly expressing tissue samples and COL1A1 highly expressing tissue samples. (B): GSVA analysis between COL1A2 lowly expressing tissue samples and COL1A2 highly expressing tissue samples. 
A

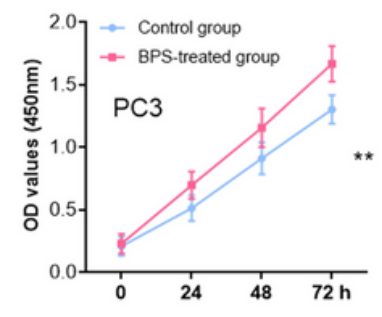

C
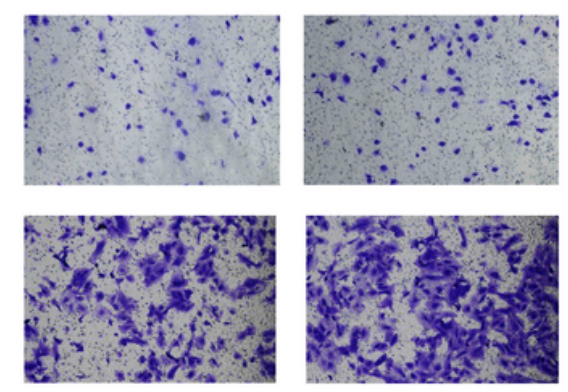

NC
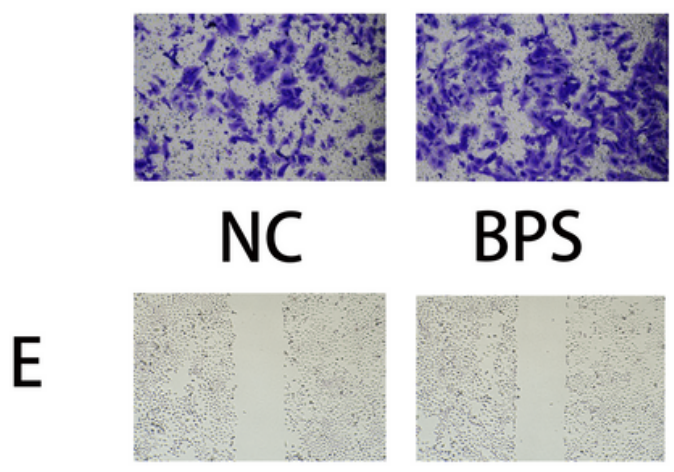

BPS
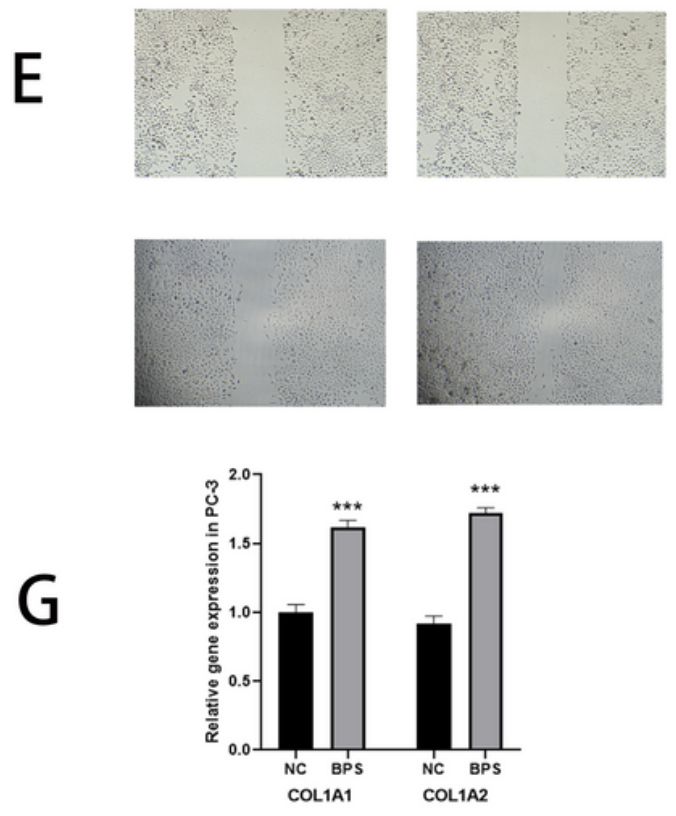

B

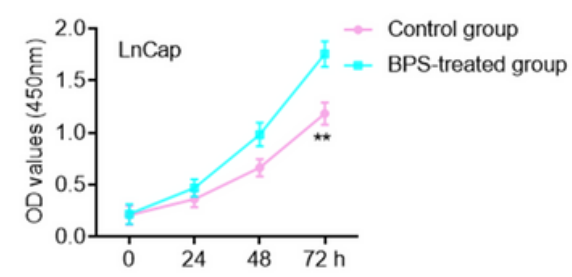

D
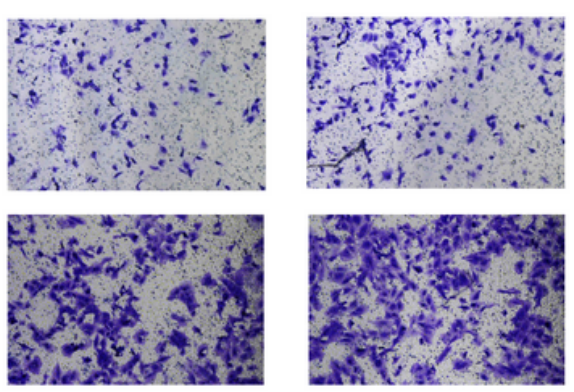

NC

$\mathrm{F}$
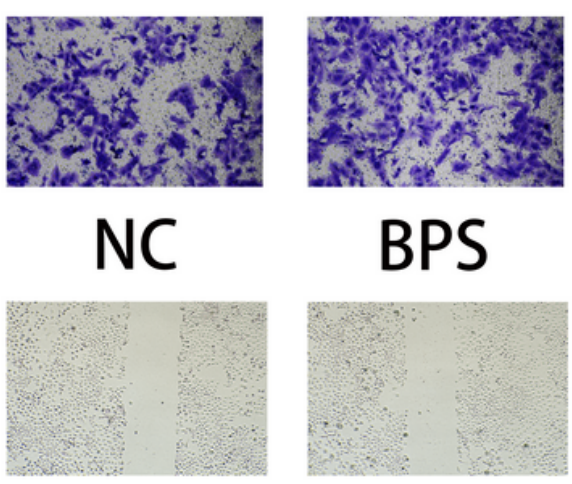

BPS
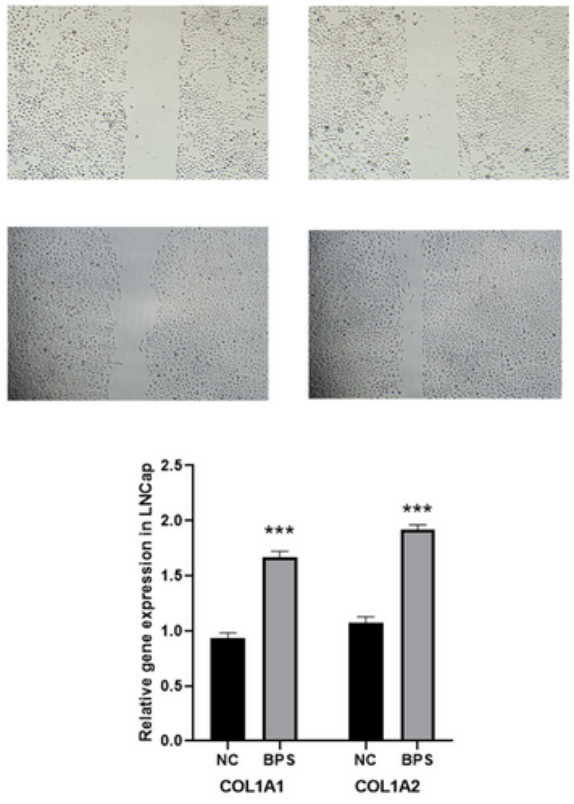

Figure 8

(A) Cell Counting Kit-8 Assay in PC-3 cells; (B) Cell Counting Kit-8 Assay in LNCap cells; (C) Transwell assay of the cells in PC-3 cells; (D) Transwell assay of the cells in LNCap cells; (E) Wound-healing assay in PC-3 cells; (F) Wound-healing assay in LNCap cells; (G) Gene expression profile of BPS treated in PC-3 cells. $(\mathrm{H})$ Gene expression profile of BPS treated in LNCap cells. 